\title{
Construction of unmodified oligonucleotide-based microarrays in the thermophilic cyanobacterium Thermosynechococcus elongatus BP-1: screening of the candidates for circadianly expressed genes
}

\author{
Ken-ichi Kucho ${ }^{1}$, Yuka Tsuchiya ${ }^{1}$, Yasuhide Okumoto ${ }^{2, \dagger}$, Manabu Harada $^{2, \dagger}$, \\ Masao Yamada ${ }^{2, \dagger}$ and Masahiro Ishiura ${ }^{1,3,4, *}$ \\ ${ }^{1}$ Center for Gene Research, Nagoya University, Furo-cho, Chikusa-ku, Nagoya 464-8602, Japan \\ ${ }^{2}$ Nippon Laser \& Electronics Lab, 20-9 Sanbonmatsu-cho, Atsuta-ku, \\ Nagoya 456-0032, Japan \\ ${ }^{3}$ Division of Biological Science, Graduate School of Science, Nagoya University, \\ Furo-cho, Chikusa-ku, Nagoya 464-8602, Japan \\ ${ }^{4}$ Bio-oriented Technology Research Advancement Institution (BRAIN), \\ Nagoya University, Furo-cho, Chikusa-ku, \\ Nagoya 464-8602, Japan
}

(Received 18 December 2004, accepted 5 January 2005)

\begin{abstract}
DNA microarrays with unmodified oligonucleotide probes are a cost-effective and high-performance alternative to cDNA microarrays. We searched every gene in the genome of the thermophilic cyanobacterium Thermosynechococcus elongatus BP-1 for 45-mer oligonucleotide probes with optimal nucleotide sequences, and found such probes in $90 \%$ of the genes. Using the probes, we constructed a microarray that represented 2,397 genes (95\% of total genes). We detected only low signals in the negative control probes whose nucleotide sequences are not contained in the $T$. elongatus genome, demonstrating that specific hybridization occurred. To evaluate the reliability of the measurements obtained by the oligonucleotide microarray, we performed microarray experiments using RNA samples from two different time points of circadianly synchronized cultures, LL2 (early subjective day) and LL14 (early subjective night). Measurements obtained from the two independent microarray hybridizations were highly concordant (correlation coefficient $[r]>0.8$ ). Northern blot analyses of 20 genes confirmed that expression changes detected by the microarrays were correct $(r=0.832)$. We identified 143 candidate clock-controlled genes whose expression levels at LL2 and LL14 were significantly different. Expression of 69 of them was enhanced at LL14 while expression of the other 74 was enhanced at LL2. The physiological functions of the genes were diverse and included metabolism, translation, transcription, membrane transport, DNA replication and repair, and cell growth and death.
\end{abstract}

Key words: circadian rhythm, microarray, Thermosynechococcus elongatus, thermophilic cyanobacteria, unmodified oligonucleotide

\section{INTRODUCTION}

Cyanobacteria are prokaryotic organisms that carry

Edited by Hideo Shinagawa

* Corresponding author. E-mail: ishiura@gene.nagoya-u.ac.jp

† Present address: Filgen, Inc., 15-1 Nakanoshima, Ohdaka-cho, Midori-ku, Nagoya 459-8001, Japan

${ }^{\dagger}$ Present address: Moritex Corporation, 1-3-3, Azamino-minami, Aoba-ku, Yokohama 225-0012, Japan out plant-like oxygen-evolving photosynthesis. Because genetic manipulation of several cyanobacterial species is easy, they are used for the study of various biological phenomena, including photosynthesis, metabolism, response to environmental stress, and circadian rhythm.

The thermophilic cyanobacterium Thermosynechococcus elongatus BP-1, which was isolated from a Japanese hot spring, grows at greater than $50^{\circ} \mathrm{C}$ (Yamaoka et al., 1978). Its proteins are highly heat-stable, which permit- 
ted X-ray analysis of the crystal structure of photosystems I (Jordan et al., 2001) and II (Zouni et al., 2001) and the circadian clock proteins KaiA (Uzumaki et al., 2004) and KaiB (our unpublished data). The three-dimensional structure of the circadian clock protein KaiC was elucidated by electron microscopy (Hayashi et al., 2003). The entire genome sequence of $T$. elongatus (2.6 Mbp) has been determined, and its genome is estimated to contain 2,524 genes (Nakamura et al., 2002). In addition, an efficient transformation procedure had been established (Onai et al., 2004a). These technical advances greatly facilitate research in the thermophilic cyanobacteria.

Circadian rhythms are self-sustaining oscillations whose period length coincides with the 24-hour day-night cycle. The rhythms are widespread and involve functions as diverse as human sleep-wake cycles and cyanobacterial nitrogen fixation. Cyanobacteria are the simplest organisms found to show circadian rhythms. kaiABC, a clock gene cluster that generates circadian rhythms, has been identified in the mesophilic cyanobacterium Synechococcus sp. strain PCC 7942 (Ishiura et al., 1998). Expression of the kaiA gene and the kaiBC operon are circadian. KaiA and KaiC proteins regulate the transcription of the kaiBC operon positively and negatively, respectively, and this feedback regulation is critical for generation of the circadian rhythms. Recently, we demonstrated by real-time bioluminescence monitoring that the circadian clock operates in T. elongatus (Onai et al., 2004b). The circadian clock of T. elongatus shows the widest temperature range $\left(30\right.$ to $\left.60^{\circ} \mathrm{C}\right)$ for the compensation of the period length among organisms investigated so far.

In the present study, we report the construction of a DNA microarray that covers almost all the $T$. elongatus genes. We used as probes unmodified oligonucleotides that allow sensitive and specific detection of transcripts at low fabrication cost (Kucho et al., 2004a). Using the microarray, we screened for candidate clock-controlled genes.

\section{MATERIALS AND METHODS}

Selection of oligonucleotide probes. We obtained nucleotide sequences of all genes contained in the T. elongatus genome (Nakamura et al., 2002) from CyanoBase (http://www.kazusa.or.jp/cyano/). First, we extracted every 45-nucleotide-span nucleotide sequence from each gene and took those with $35-55 \%$ GC and $<20 \% \mathrm{~T}$ using Perl scripts (Wall et al., 2002). Second, we used the BLAST program (Altschul et al., 1997) with word size set at 7 and gap penalty at -3 to reject nucleotide sequences that showed $>70 \%$ similarity to other genes in the genome. Third, we used the RNAfold program (Zuker and Stiegler, 1981; http://www.tbi.univie.ac.at/\%7Eivo/ $\mathrm{RNA} /$ ) to reject nucleotide sequences that showed $<-10.5$ kcal/mol free energy for the predicted secondary structure. Fourth, we used the Tandem Repeat Finder program (Benson, 1999; http://c3.biomath.mssm.edu/trf.html) to reject nucleotide sequences that contained repetitive sequences. We selected the surviving sequence with the lowest similarity to other genes in the genome as a probe for each gene. When nucleotide sequences satisfying all four criteria were not found, we selected a probe that satisfied as many criteria as possible and was the least similar to other genes in the genome.

Construction of microarray. We constructed the microarrays using a DNA Microarray Kit (Nippon Laser \& Electronics Lab, Japan) according to the manufacturer's instructions. Unmodified oligonucleotide probes were synthesized chemically, purified by gel filtration (Nihon Gene Research Laboratories Inc.), and robotically spotted onto glass slides with a Gtmas Stamp II microarray spotter (Nippon Laser \& Electronics Lab, Japan). The concentration of oligonucleotide solutions used for spotting was $3 \mu \mathrm{M}$. 45-mer oligonucleotides with artificial nucleotide sequences that show $<58 \%$ similarity to any $T$. elongatus genes were spotted as negative control probes. We fragmented the genomic DNA of T. elongatus by sonication and spotted $0.03,0.1,0.3,1,3,10$, and $30 \mathrm{ng} / \mu \mathrm{l}$ solutions. DNAs were immobilized on glass slides by UV cross-linking at $60 \mathrm{~mJ} / \mathrm{cm}^{2}$. Hybridizationready microarrays were stored in a desiccator at room temperature.

Strain and culture conditions. We grew wild-type $T$. elongatus (Yamaoka et al., 1978) at $50^{\circ} \mathrm{C}$ under constant light from white fluorescent lamps at $38 \mu \mathrm{mol} \mathrm{m} \mathrm{mec}^{-1}$ (hereafter called LL conditions) in BG-11 liquid medium (Rippka et al., 1979) with bubbling of air containing 5\% (v/v) $\mathrm{CO}_{2}$. We subjected the cells to $12 \mathrm{~h}$ of darkness to synchronize the circadian clock, and transferred them back to LL. We collected cells for RNA isolation at $2 \mathrm{~h}$ (LL2) and $14 \mathrm{~h}$ (LL14) after the transfer.

Microarray experiments. We isolated total RNAs from two independent cultures by the hot-phenol method (Kucho et al., 2004a) and purified them using the SV total RNA isolation system (Promega, WI, USA). We used a mixture of the total RNAs from the two cultures for labeling reactions. We synthesized fluorescence-labeled cDNA by direct incorporation of Cy3-dUTP or Cy5-dUTP (Amersham Bioscience, NJ, USA) during random-primed reverse transcription, using $5.9 \mu \mathrm{g}$ total RNA and an RNA fluorescence labeling core kit (M-MLV version 2.0, TaKaRa, Japan). We prehybridized the microarray for 1 $\mathrm{h}$ at $42^{\circ} \mathrm{C}$ in a solution containing $5 \times \mathrm{SSC}(1 \times \mathrm{SSC}$ is 0.15 $\mathrm{M} \mathrm{NaCl}, 0.015 \mathrm{M}$ sodium citrate), $0.1 \%$ sodium lauryl sulfate (SDS), and $10 \mathrm{mg} / \mathrm{ml}$ bovine serum albumin. We washed the microarray at room temperature in distilled 
water 3 times for $1 \mathrm{~min}$, rinsed it in 2-propanol, and dried it by centrifugation at $150 \times \mathrm{g}$ for $2 \mathrm{~min}$. We performed hybridization for $16 \mathrm{~h}$ at $42^{\circ} \mathrm{C}$ in $12-\mu \mathrm{L}$ solution containing $5 \times$ SSC, $0.1 \%$ SDS, $30 \%$ formamide, and heat-denatured labeled cDNA. We then washed the microarray at room temperature with $2 \times$ SSC containing $0.1 \%$ SDS for $4 \mathrm{~min}$, with $0.1 \times \mathrm{SSC}$ containing $0.1 \%$ SDS for $4 \mathrm{~min}$, and 3 times with $0.1 \times \mathrm{SSC}$ for $1 \mathrm{~min}$. We dried the microarray by centrifugation. We obtained fluorescence images of $\mathrm{Cy} 3$ and $\mathrm{Cy} 5$ dye channels using a GenePix 4000B scanner (Axon Instruments, CA, USA).

Data analysis. We used GenePix Pro 5.0 software (Axon Instruments) to determine the signal intensity of each spot and its local background. We calculated net signal intensity by subtracting the median signal intensity of all pixels within the local background area from the median signal intensity of all pixels within the spot area. We visually confirmed the correct recognition of all spot areas by the automatic alignment function of the GenePix Pro. We flagged spots and did not use them for data analysis when any of following occurred: (i) the GenePix Pro did not find the spot area automatically, (ii) the net signal intensity was $\leq 0$, (iii) the percentage of saturated pixels in the spot area was $\geq 25$, and (iv) severe noise was present. We normalized biases in signal intensity between the two fluorescent dye channels in a microarray by locally weighted linear regression analysis (lowess normalization) (Yang et al., 2002) using MIDAS software (http://www.tigr.org/software/tm4/midas.html). For all normalization, we set the smoothing parameter to 0.33. Microarray data were deposited in the Gene Expression Omnibus (GEO) (http://www.ncbi.nlm.nih.gov/geo/) under accession number GSE2114.

We used the Student $t$-test (Sanders, 1995) to evaluate the significance of difference in expression level between LL2 and LL14 ( $\log _{2}[$ LL14 / LL2]). We controlled type I errors (false positives) by the method of Holm (1979) and considered $P<0.05$ as significant.

We used KEGG database (Kanehisa et al., 2002; http:// www.genome.jp/dbget-bin/get_htext?T.elongatus.kegg) to assign names, annotations and functional categories of genes (Tables 1 and 2). Functional categories of some genes were unassigned in KEGG database but they were apparent from our knowledge. In such cases, we discussed the functions in the text (e.g. tllo855, tllo854, tlr2302, and tlr0873).

Northern blot analysis. We separated total RNA (5 $\mu \mathrm{g})$ by electrophoresis on a denaturing agarose gel containing formaldehyde and blotted it to a Biodyne B nylon membrane (Pall, NY, USA) (Sambrook and Russell, 2001). We electrophoresed each RNA sample in three lanes. We end-labeled 45-mer oligonucleotides complementary to the microarray probes with $\left[\gamma_{-}{ }^{32} \mathrm{P}\right]$ ATP, and hybridized them in ExpressHyb Hybridization Solution (Clontech, CA, USA) at $60^{\circ} \mathrm{C}$ for $1 \mathrm{~h}$. We washed the membranes in $0.1 \times \mathrm{SSC}$ containing $0.1 \% \mathrm{SDS}$ at $50^{\circ} \mathrm{C}$. We quantified band signal intensities using a BAS2000 image analyzer (Fujifilm, Tokyo, Japan). We used the Student $t$-test (Sanders, 1995) corrected by the Holm method (Holm, 1979) to evaluate differences in expression level between LL2 and LL14 and considered $P<0.05$ significant.

\section{RESULTS AND DISCUSSION}

Construction of unmodified oligonucleotide-based microarray. In our previous study, we demonstrated that DNA microarrays with unmodified oligonucleotide probes detected transcripts with sensitivity and specificity when appropriate oligonucleotide sequences were selected (Kucho et al., 2004a). We applied those selection criteria to 2,521 T. elongatus genes. Three ribosomal RNA genes were omitted because their expression levels are extremely high and cannot be precisely quantified by microarray analysis. 2,264 genes (90\%) contained at least one optimal probe sequence that satisfied all the selection criteria (see MATERIALS AND METHODS). Such probes could not be designed for 257 (10\%) of the genes. Most probes in those genes showed an inappropriate base composition (129 genes) or high sequence similarity to other genes in the genome (105 genes). The latter were members of gene families. In particular, four reverse transcriptase genes shared a completely identical sequence, and 76 putative transposase genes shared 86 to $100 \%$ sequence similarity. We therefore included in the microarray a representative gene for each of the gene families.

Using the oligonucleotide probes, we constructed a microarray that represented 2,397 genes (95\% of total genes contained in the genome). The microarray also contained 39 control probes (32 negative controls and 7 serial dilutions of genomic DNA). We did not include genes of $\leq 100$ bp because such short transcripts are removed during RNA purification. We spotted all the oligonucleotide probes in duplicate. Fig. 1 shows a typical hybridization image of the microarray. The fluorescent signals were uniform and homogeneous, and signal/ background contrast was high.

Specificity of hybridization signals. To evaluate specificity of hybridization, we compared signal intensities between $T$. elongatus genes and negative control probes whose nucleotide sequences did not show significant similarity to any T. elongatus genes. As shown in Fig. 2, most negative control probes exhibited very low signals while $T$. elongatus gene probes showed a wide range of signal intensities. This result suggests that sequence-specific hybridizations occurred on the oligonu- 
cleotide microarray.

Comparison of gene expression in $T$. elongatus at two different circadian times. We compared gene expression levels at two different circadian times - LL2 (early subjective day) and LL14 (early subjective night). The experiment had two purposes: (i) to evaluate the reliability of the measurements obtained by the oligonucleotide microarray, and (ii) to screen for candidate clockcontrolled genes. We performed two sets of microarray experiments. In one set, two microarrays were hybrid- ized with a mixture of solutions containing LL2 cDNA labeled with $\mathrm{Cy} 3$ and LL14 cDNA labeled with Cy5. In the other set, the fluorescent dyes were swapped. The expression ratios (LL14 / LL2) of genes obtained by the two independent microarray hybridizations were similar (correlation coefficient $[r]=0.853$ and 0.818 ) (Fig. 3). Fig. 4 shows the mean expression ratios obtained by the four microarray hybridizations. The microarray contained spots of serial dilutions of $T$. elongatus genomic DNA. As expected, all the spots showed a signal ratio near 1.0, suggesting that biases in signal intensities

Table 1. Genes enhanced in the early subjective day (LL2).

\begin{tabular}{|c|c|c|c|c|}
\hline $\mathrm{ID}^{\mathrm{a}}$ & $\mathrm{LL}_{14 / \mathrm{LL} 2}{ }^{\mathrm{b}}$ & Gene $^{\mathrm{c}}$ & Annotation $^{c}$ & Functional category $^{\mathrm{c}}$ \\
\hline$t \operatorname{lr} 0605$ & $0.25(0.20-0.32)$ & & $\begin{array}{l}\text { 4-Diphosphocytidyl-2C-methyl-D-erythritol } \\
\text { synthase }\end{array}$ & Lipid metabolism \\
\hline tlr0873 & $0.26(0.23-0.30)$ & $h \operatorname{spA}$ & 16.6 kDa small heat shock protein molecular chaperon & Unassigned \\
\hline tll0856 & $0.36(0.34-0.39)$ & & Hypothetical protein & Unassigned \\
\hline tll0165 & $0.42(0.37-0.48)$ & & Hypothetical protein & Unassigned \\
\hline $\operatorname{tll1670}$ & $0.42(0.38-0.46)$ & & Hypothetical protein & Unassigned \\
\hline tsl0163 & $0.44(0.41-0.48)$ & & Hypothetical protein & Unassigned \\
\hline $\operatorname{tlr} 2298$ & $0.45(0.41-0.50)$ & & Hypothetical protein & Unassigned \\
\hline$t l l 2470$ & $0.45(0.38-0.54)$ & & Dps family DNA-binding stress response protein & Replication and repair \\
\hline $\operatorname{tll1651}$ & $0.48(0.41-0.57)$ & & Hypothetical protein & Unassigned \\
\hline$t l l 1652$ & $0.48(0.43-0.53)$ & & Unknown protein & Unassigned \\
\hline $\operatorname{tl} 2301$ & $0.49(0.42-0.59)$ & & Hypothetical protein & Unassigned \\
\hline$t \operatorname{lr} 0226$ & $0.49(0.45-0.53)$ & & Pyruvate formate lyase activating enzyme & Unassigned \\
\hline $\operatorname{tll1316}$ & $0.49(0.41-0.57)$ & & 6-Phosphofructokinase & Carbohydrate metabolism \\
\hline $\operatorname{tsr} 1332$ & $0.50(0.46-0.55)$ & & Hypothetical protein & Unassigned \\
\hline $\operatorname{tll0855}$ & $0.50(0.43-0.58)$ & & Similar to allophycocyanin beta-18 subunit & Unassigned \\
\hline$t \operatorname{lr} 1060$ & $0.52(0.48-0.56)$ & & Unknown protein & Unassigned \\
\hline $\operatorname{tll0854}$ & $0.53(0.48-0.60)$ & & Similar to allophycocyanin alpha subunit & Unassigned \\
\hline $\operatorname{tlr} 2302$ & $0.53(0.49-0.57)$ & & Probable ferredoxin & Unassigned \\
\hline $\operatorname{tr} 0436$ & $0.54(0.49-0.61)$ & & Mannose-1-phosphate guanyltransferase & Carbohydrate metabolism \\
\hline $\operatorname{tlr} 1372$ & $0.54(0.50-0.58)$ & & Unknown protein & Unassigned \\
\hline $\operatorname{tlr} 1526$ & $0.54(0.47-0.63)$ & & Hypothetical protein & Unassigned \\
\hline$t l l 0513$ & $0.55(0.48-0.63)$ & & Iron binding protein component of $\mathrm{ABC}$ iron transporter & Unassigned \\
\hline $\operatorname{tr} 0319$ & $0.56(0.50-0.63)$ & & Hypothetical protein & Unassigned \\
\hline $\operatorname{tlr} 1257$ & $0.56(0.52-0.60)$ & & Hypothetical protein & Unassigned \\
\hline$t \operatorname{lr0965}$ & $0.58(0.51-0.67)$ & & Unknown protein & Unassigned \\
\hline $\operatorname{tll1688}$ & $0.58(0.52-0.65)$ & rps2 & $30 \mathrm{~S}$ ribosomal protein $\mathrm{S} 2$ & Translation \\
\hline $\operatorname{tll0515}$ & $0.58(0.54-0.61)$ & & Hypothetical protein & Unassigned \\
\hline$t \operatorname{lr} 0227$ & $0.59(0.53-0.65)$ & & $\begin{array}{l}\text { Alcohol dehydrogenase / acetaldehyde } \\
\text { dehydrogenase }\end{array}$ & $\begin{array}{l}\text { Carbohydrate metabolism, Lipid } \\
\text { metabolism, Amino acid metabolism }\end{array}$ \\
\hline $\operatorname{tll0185}$ & $0.59(0.53-0.65)$ & groEL-1 & $60-\mathrm{kD}$ chaperonin 1 & Folding, sorting and degradation \\
\hline$t \operatorname{lr} 1333$ & $0.60(0.55-0.65)$ & & Hypothetical protein & Unassigned \\
\hline $\operatorname{tsr} 1145$ & $0.60(0.56-0.64)$ & & Hypothetical protein & Unassigned \\
\hline $\operatorname{tlr} 1425$ & $0.60(0.55-0.64)$ & & Hypothetical protein & Unassigned \\
\hline$t l l 2200$ & $0.60(0.55-0.65)$ & & Hypothetical protein & Unassigned \\
\hline tll1781 & $0.61(0.56-0.67)$ & & Unknown protein & Unassigned \\
\hline
\end{tabular}




\begin{tabular}{|c|c|c|c|c|}
\hline \multicolumn{5}{|c|}{ Continued } \\
\hline tllo007 & $0.61(0.53-0.70)$ & & Cellulose synthase & Unassigned \\
\hline $\operatorname{tlr1565}$ & $0.62(0.55-0.70)$ & & Hypothetical protein & Unassigned \\
\hline tll0839 & $0.62(0.56-0.69)$ & & Hypothetical protein & Unassigned \\
\hline tlr0694 & $0.62(0.57-0.66)$ & pntA & $\begin{array}{l}\text { Pyridine nucleotide transhydrogenase alpha } \\
\text { subunit }\end{array}$ & Metabolism of cofactors and vitamins \\
\hline$t \operatorname{lr} 2270$ & $0.62(0.56-0.67)$ & & Hypothetical protein & Unassigned \\
\hline$t \operatorname{lr} 2303$ & $0.63(0.58-0.69)$ & & Hypothetical protein & Unassigned \\
\hline$t \operatorname{tr} 2286$ & $0.63(0.56-0.70)$ & $\operatorname{tr} x M 1$ & Thioredoxin M & Unassigned \\
\hline tsr0695 & $0.64(0.59-0.70)$ & & $\begin{array}{l}\text { Similar to pyridine nucleotide transhydrogenase } \\
\text { alpha subunit }\end{array}$ & Metabolism of cofactors and vitamins \\
\hline$t \operatorname{lr} 1424$ & $0.64(0.57-0.72)$ & & Unknown protein & Unassigned \\
\hline tlr0419 & $0.64(0.58-0.70)$ & & Fibrillin & Unassigned \\
\hline tllo747 & $0.64(0.57-0.72)$ & & Dihydroneopterin aldolase & Metabolism of cofactors and vitamins \\
\hline tlr0829 & $0.65(0.59-0.73)$ & & Hypothetical protein & Unassigned \\
\hline tll0248 & $0.65(0.62-0.69)$ & & Hypothetical protein & Unassigned \\
\hline tlr0598 & $0.65(0.60-0.70)$ & & Putative glutaminase & $\begin{array}{l}\text { Energy metabolism, Amino acid } \\
\text { metabolism, Metabolism of other } \\
\text { amino acids }\end{array}$ \\
\hline tlr0095 & $0.65(0.57-0.73)$ & rpl6 & $50 \mathrm{~S}$ ribosomal protein $\mathrm{L} 6$ & Translation \\
\hline$t \operatorname{lr} 1649$ & $0.67(0.61-0.74)$ & & $\mathrm{ABC}$ transporter ATP-binding protein & Membrane transport \\
\hline tll1149 & $0.68(0.64-0.73)$ & & $\begin{array}{l}\text { Putative transcriptional regulators, homologs of } \\
\text { Bvg accessory factor }\end{array}$ & Transcription \\
\hline tlr0096 & $0.69(0.65-0.73)$ & rpl18 & 50S ribosomal protein L18 & Translation \\
\hline $\operatorname{tlr} 1879$ & $0.69(0.63-0.75)$ & & Hypothetical protein & Unassigned \\
\hline tlr0291 & $0.70(0.67-0.74)$ & & Hypothetical protein & Unassigned \\
\hline tsl2083 & $0.70(0.65-0.75)$ & & Unknown protein & Unassigned \\
\hline$t \operatorname{lr} 2341$ & $0.70(0.66-0.74)$ & pilM & Probable fimbrial assembly protein PilM & Folding, sorting and degradation \\
\hline$t \operatorname{lr} 1595$ & $0.71(0.65-0.77)$ & & Unknown protein & Unassigned \\
\hline$t s r 2448$ & $0.72(0.67-0.78)$ & & Hypothetical protein & Unassigned \\
\hline tllo746 & $0.72(0.65-0.79)$ & & Hypothetical protein & Unassigned \\
\hline tsl2428 & $0.72(0.68-0.76)$ & & Hypothetical protein & Unassigned \\
\hline$t l l 2347$ & $0.73(0.69-0.78)$ & $\operatorname{chlL}$ & $\begin{array}{l}\text { Light-independent protochlorophyllide reductase } \\
\text { iron protein subunit }\end{array}$ & Metabolism of cofactors and vitamins \\
\hline $\operatorname{tlr} 1215$ & $0.74(0.69-0.78)$ & & Two-component sensor histidine kinase & Unassigned \\
\hline tlr0924 & $0.75(0.70-0.82)$ & & Hypothetical protein & Unassigned \\
\hline tlr0111 & $0.75(0.71-0.79)$ & & Hypothetical protein & Unassigned \\
\hline tll0130 & $0.75(0.70-0.80)$ & menD & Menaquinone biosynthesis protein & Metabolism of cofactors and vitamins \\
\hline tlr0977 & $0.75(0.69-0.80)$ & & Unknown protein & Unassigned \\
\hline tll1591 & $0.76(0.72-0.80)$ & & Glycosyl transferase & Unassigned \\
\hline tlr2085 & $0.77(0.74-0.80)$ & & Hypothetical protein & Unassigned \\
\hline tll1647 & $0.77(0.73-0.81)$ & & Hypothetical protein & Unassigned \\
\hline tll1195 & $0.77(0.72-0.82)$ & & Hypothetical protein & Unassigned \\
\hline tlr0092 & $0.79(0.74-0.85)$ & $r p l 24$ & 50 S ribosomal protein L24 & Translation \\
\hline$t l l 1487$ & $0.82(0.78-0.86)$ & & Hypothetical protein & Unassigned \\
\hline tll1189 & $0.85(0.82-0.88)$ & cpmA & Circadian phase modifier CpmA homolog & Unassigned \\
\hline$t \operatorname{lr} 1961$ & $0.85(0.82-0.88)$ & $c p c E$ & Phycocyanin alpha-subunit phycocyanobilin lyase & Energy metabolism \\
\hline
\end{tabular}

${ }^{a}$ ID for genes is according to Nakamura et al. (2002). ${ }^{b}$ Mean expression ratio obtained by the four microarray experiments. Parentheses contain the range of the expression ratio in consideration of SD. ${ }^{\mathrm{c}}$ Gene symbols, annotations, functional categories are according to KEGG (http://www.genome.jp/dbget-bin/get_htext?T.elongatus.kegg). 
between the two fluorescent dye channels were correctly normalized. Using a statistical test, we identified 143 genes whose LL2 and LL14 expression levels were significantly different. The expression of 69 genes was enhanced in the early subjective night (LL14 / LL2 > 1), and the expression of the remaining 74 genes was enhanced in the early subjective day (LL14 / LL2 < 1). We confirmed the expression changes of 20 genes by Northern blot analysis (Fig. 5). Concordance of the expression ratios obtained by the two different methods was high $(r=0.832)$. The expression changes of $14(70 \%)$ genes detected by Northern blot analysis were statistically significant.

Tables 1 and 2 list the genes whose expression was enhanced in the early subjective day (LL2) or early subjective night (LL14). In considering the possible importance of these genes on cellular physiology, we used a functional category list in KEGG (http://www.genome.jp/

Table 2. Genes enhanced in the early subjective night (LL14).

\begin{tabular}{|c|c|c|c|c|}
\hline $\mathrm{ID}^{\mathrm{a}}$ & $\mathrm{LL}_{14 / \mathrm{LL}}{ }^{\mathrm{b}}$ & Gene $^{\mathrm{c}}$ & Annotation $^{\mathrm{c}}$ & Functional category $^{\mathrm{c}}$ \\
\hline$t \operatorname{lr} 1288$ & $4.08(3.74-4.46)$ & $n d h H$ & NADH dehydrogenase subunit 7 & Energy metabolism \\
\hline $\operatorname{tlr} 1643$ & $2.97(2.57-3.42)$ & $a c c D$ & Acetyl-CoA carboxylase beta subunit & $\begin{array}{l}\text { Carbohydrate metabolism, Lipid } \\
\text { metabolism, Biosynthesis of secondary metabolites }\end{array}$ \\
\hline$t l l 1269$ & $2.72(2.21-3.35)$ & & Hypothetical protein & Unassigned \\
\hline $\operatorname{tlr} 0636$ & $2.69(2.29-3.16)$ & & Hypothetical protein & Unassigned \\
\hline tll0045 & $2.68(2.24-3.20)$ & $n d h B$ & NADH dehydrogenase subunit 2 & Energy metabolism \\
\hline $\operatorname{tlr} 1289$ & $2.65(2.40-2.91)$ & & Hypothetical protein & Unassigned \\
\hline $\operatorname{tlr} 0325$ & $2.44(2.11-2.81)$ & $\operatorname{ser} A$ & D-3-Phosphoglycerate dehydrogenase & Amino acid metabolism \\
\hline $\operatorname{tr} 0667$ & $2.27(2.08-2.49)$ & $n d h A$ & NADH dehydrogenase subunit 1 & Energy metabolism \\
\hline tsl1865 & $2.27(1.80-2.86)$ & & Transcriptional regulator & Unassigned \\
\hline $\operatorname{tll1268}$ & $2.25(1.95-2.60)$ & & Hypothetical protein & Unassigned \\
\hline $\operatorname{tsr} 0473$ & $2.22(2.00-2.47)$ & & Probable bacterioferritin comigratory protein & Unassigned \\
\hline$t s l 2208$ & $2.15(1.91-2.41)$ & & CAB/ELIP/HLIP superfamily protein & Unassigned \\
\hline $\operatorname{tlr} 1088$ & $2.13(1.90-2.39)$ & & Probable flavoprotein & Unassigned \\
\hline $\operatorname{tlr} 1819$ & $2.13(1.89-2.40)$ & $n d h D 2$ & $\mathrm{NADH}$ dehydrogenase subunit 4 & Energy metabolism \\
\hline$t l l 0220$ & $2.10(1.86-2.36)$ & & Hypothetical protein & Unassigned \\
\hline $\operatorname{tlr} 0472$ & $2.01(1.72-2.34)$ & & Hypothetical protein & Unassigned \\
\hline $\operatorname{tr} 0668$ & $1.98(1.85-2.13)$ & $n d h I$ & NADH dehydrogenase I subunit I & Energy metabolism \\
\hline $\operatorname{tlr} 1876$ & $1.95(1.80-2.12)$ & & Hypothetical protein & Unassigned \\
\hline tll0734 & $1.94(1.78-2.12)$ & $f t s H$ & Cell division protein & $\begin{array}{l}\text { Folding, sorting and degradation, Cell } \\
\text { growth and death }\end{array}$ \\
\hline $\operatorname{tll1587}$ & $1.90(1.78-2.04)$ & & Probable GTP-binding protein & Unassigned \\
\hline $\operatorname{tlr} 1875$ & $1.87(1.79-1.96)$ & & Hypothetical protein & Unassigned \\
\hline$t \operatorname{lr} 1709$ & $1.85(1.73-1.98)$ & & Hypothetical protein & Unassigned \\
\hline$t l l 0044$ & $1.78(1.67-1.90)$ & & Hypothetical protein & Unassigned \\
\hline$t \operatorname{lr} 0637$ & $1.77(1.53-2.06)$ & & Hypothetical protein & Unassigned \\
\hline$t l l 0946$ & $1.75(1.55-1.98)$ & $c c m K$ & $\begin{array}{l}\text { Carbon dioxide concentrating mechanism } \\
\text { protein }\end{array}$ & Unassigned \\
\hline $\operatorname{tlr} 1757$ & $1.74(1.62-1.88)$ & $s d s$ & Solanesyl diphosphate synthase & Unassigned \\
\hline $\operatorname{tll0490}$ & $1.74(1.49-2.02)$ & $y c f 24$ & ABC transporter subunit & Unassigned \\
\hline$t l l 2067$ & $1.73(1.64-1.82)$ & & Hypothetical protein & Unassigned \\
\hline $\operatorname{tll1559}$ & $1.72(1.55-1.91)$ & & Probable phosphoribosyltransferase & Unassigned \\
\hline $\operatorname{tlr} 1749$ & $1.68(1.53-1.84)$ & fus & Translation elongation factor EF-G & Translation \\
\hline $\operatorname{tll0596}$ & $1.65(1.53-1.77)$ & & Probable heme d1 biosynthesis protein NirJ & Unassigned \\
\hline $\operatorname{tlr} 1747$ & $1.64(1.56-1.73)$ & rps 12 & $30 \mathrm{~S}$ ribosomal protein $\mathrm{S} 12$ & Translation \\
\hline $\operatorname{tll1842}$ & $1.63(1.45-1.85)$ & & Putative arsenical pump-driving ATPase & Unassigned \\
\hline $\operatorname{tr} 0705$ & $1.63(1.53-1.74)$ & $n d h K$ & NADH dehydrogenase subunit & Energy metabolism \\
\hline tllo772 & $1.63(1.50-1.77)$ & & Hypothetical protein & Unassigned \\
\hline
\end{tabular}




\begin{tabular}{|c|c|c|c|c|}
\hline \multicolumn{5}{|c|}{ Continued } \\
\hline tlr0982 & $1.62(1.47-1.78)$ & $t h r C$ & Threonine synthase & $\begin{array}{l}\text { Amino acid metabolism, Metabolism of } \\
\text { cofactors and vitamins }\end{array}$ \\
\hline tllo944 & $1.61(1.43-1.81)$ & ccmM & Carbon dioxide concentrating mechanism protein & Unassigned \\
\hline tlr0547 & $1.56(1.45-1.67)$ & & Unknown protein & Unassigned \\
\hline tll2117 & $1.55(1.37-1.74)$ & & Probable transcriptional regulator & Unassigned \\
\hline tlr0147 & $1.53(1.40-1.67)$ & rps4 & $30 \mathrm{~S}$ ribosomal protein $\mathrm{S} 4$ & Translation \\
\hline tlr0818 & $1.52(1.35-1.71)$ & & Deoxyhypusine synthase & Unassigned \\
\hline tll1313 & $1.52(1.39-1.65)$ & & Hypothetical protein & Unassigned \\
\hline tlr0508 & $1.51(1.37-1.66)$ & & Trigger factor & Folding, sorting and degradation \\
\hline tll2264 & $1.50(1.40-1.61)$ & & Unknown protein & Unassigned \\
\hline tllo642 & $1.48(1.39-1.58)$ & rров & RNA polymerase beta subunit & Nucleotide metabolism, Transcription \\
\hline tsr1584 & $1.47(1.37-1.59)$ & & Hypothetical protein & Unassigned \\
\hline tsr0983 & $1.46(1.33-1.60)$ & & Hypothetical protein & Unassigned \\
\hline tsl1386 & $1.44(1.35-1.54)$ & $p s b H$ & Photosystem II PsbH protein & Energy metabolism \\
\hline $\operatorname{tll1826}$ & $1.44(1.36-1.52)$ & rps14 & $30 \mathrm{~S}$ ribosomal protein $\mathrm{S} 14$ & Translation \\
\hline $\operatorname{tll1511}$ & $1.43(1.31-1.57)$ & chlI & Magnesium-chelatase subunit & Metabolism of cofactors and vitamins \\
\hline tsl1385 & $1.38(1.27-1.49)$ & & Sec-independent protein translocase protein TatA & Folding, sorting and degradation \\
\hline tlr0670 & $1.38(1.31-1.45)$ & $n d h E$ & NADH dehydrogenase subunit $4 \mathrm{~L}$ & Energy metabolism \\
\hline tll1466 & $1.37(1.26-1.50)$ & & Glyceraldehyde-3-phosphate dehydrogenase & Carbohydrate metabolism \\
\hline tlr0320 & $1.37(1.26-1.48)$ & & Unknown protein & Unassigned \\
\hline$t \operatorname{lr} 2443$ & $1.35(1.25-1.45)$ & $y c f 50$ & Hypothetical protein & Unassigned \\
\hline $\operatorname{tll1384}$ & $1.34(1.25-1.43)$ & pth & Peptidyl-tRNA hydrolase & Translation \\
\hline $\operatorname{tlr} 1918$ & $1.34(1.26-1.42)$ & & Hypothetical protein & Unassigned \\
\hline tsr0293 & $1.33(1.25-1.43)$ & $\sec E$ & Preprotein translocase SecE subunit & Folding, sorting and degradation \\
\hline tllo043 & $1.32(1.25-1.40)$ & gap1 & Glyceraldehyde 3-phosphate dehydrogenase & Carbohydrate metabolism \\
\hline $\operatorname{tll1463}$ & $1.32(1.22-1.42)$ & & Hypothetical protein & Unassigned \\
\hline $\operatorname{tlr} 1854$ & $1.29(1.26-1.32)$ & & Probable glycosyl transferase & Unassigned \\
\hline $\operatorname{tlr} 1661$ & $1.27(1.21-1.34)$ & & Hypothetical protein & Unassigned \\
\hline tlr0376 & $1.27(1.19-1.35)$ & $f b a A$ & Class II fructose-bisphosphate aldolase & $\begin{array}{l}\text { Carbohydrate metabolism, Energy } \\
\text { metabolism }\end{array}$ \\
\hline tlr0783 & $1.25(1.19-1.32)$ & & Integral membrane signal transducer protein & Unassigned \\
\hline $\operatorname{tlr} 1474$ & $1.25(1.19-1.31)$ & & Hypothetical protein & Membrane transport \\
\hline tll1938 & $1.23(1.16-1.30)$ & & Hypothetical protein & Unassigned \\
\hline tll2464 & $1.22(1.16-1.29)$ & $p s b Z$ & Photosystem II $11 \mathrm{kD}$ protein & Energy metabolism \\
\hline $\operatorname{tlr} 1279$ & $1.17(1.13-1.21)$ & rps6 & $30 \mathrm{~S}$ ribosomal protein $\mathrm{S} 6$ & Translation \\
\hline tlr0334 & $1.16(1.12-1.21)$ & thiC & Thiamine biosynthesis protein & Metabolism of cofactors and vitamins \\
\hline
\end{tabular}

${ }^{a}$ ID for genes is according to Nakamura et al. (2002). ${ }^{b}$ Mean expression ratio obtained by the four microarray experiments. Parentheses contain the range of the expression ratio in consideration of SD. ${ }^{\mathrm{c}} \mathrm{Gene}$ symbols, annotations, functional categories are according to KEGG (http://www.genome.jp/dbget-bin/get_htext?T.elongatus.kegg).

dbget-bin/get_htext?T.elongatus.kegg). Of the 143 possible clock-controlled genes, 53\% were assigned predicted annotations. The physiological roles of the candidate clock-controlled genes were diverse (Fig. 6).

Genes with expression enhanced in the early subjective day. Expression of several genes associated with photosynthesis were enhanced in the early subjective day (Table 1). They included three genes encoding subunits of the light-harvesting phycobilisome complex (tll0855, tll0854, and tlr1961 [cpcE]), the chlL gene (tll2347) encoding the light-independent protochlorophyllide reductase that is involved in chlorophyll synthesis, and a gene encoding ferredoxin (tlr2302), which is a photosystem electron carrier. Two chaperonin genes, $h s p A$ and groEL-1 (tlr0873 and tll0185), were also activated in the early subjective day. The chaperonin gene dnaK shows circadian rhythm and peaks just before the subjective morning in the cyanobacterium Synechocystis sp. strain PCC 6803 (Aoki et al., 1995). Circadian regula- 

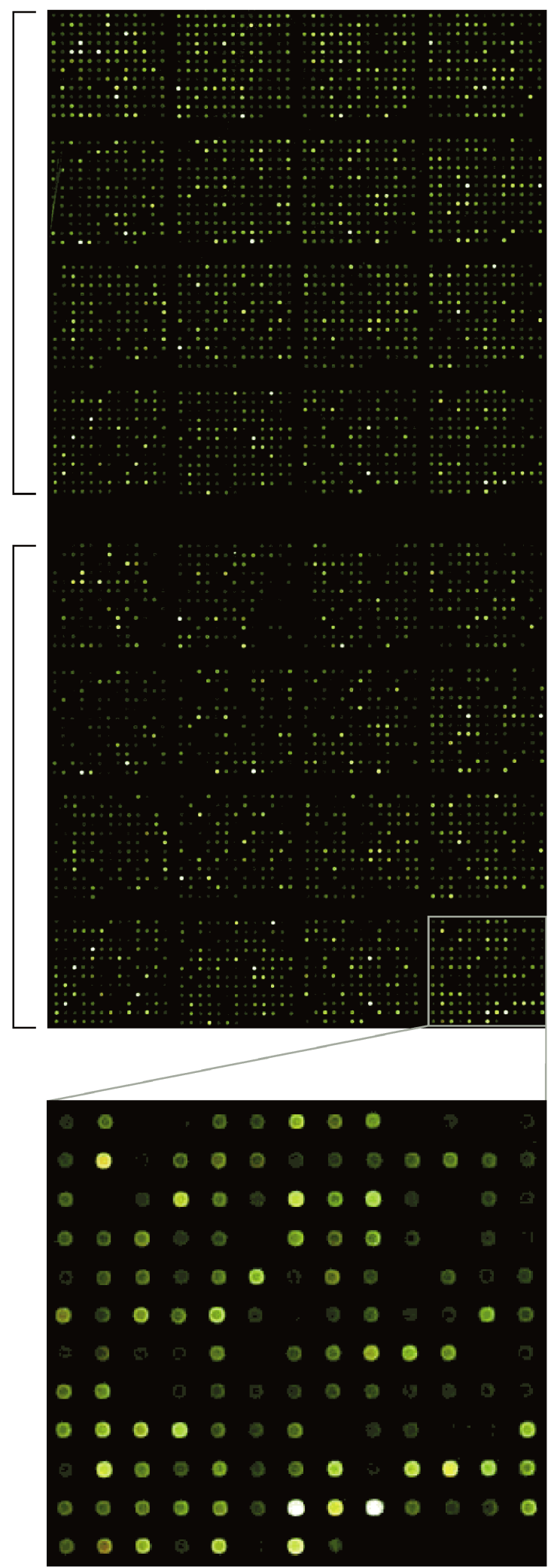

Fig. 1. A typical hybridization image of the microarray. Brackets represent duplicates. The lower image is a magnified view of a part of the microarray. LL2 and LL14 cDNAs were labeled with $\mathrm{Cy} 5$ (red) and $\mathrm{Cy} 3$ (green), respectively.

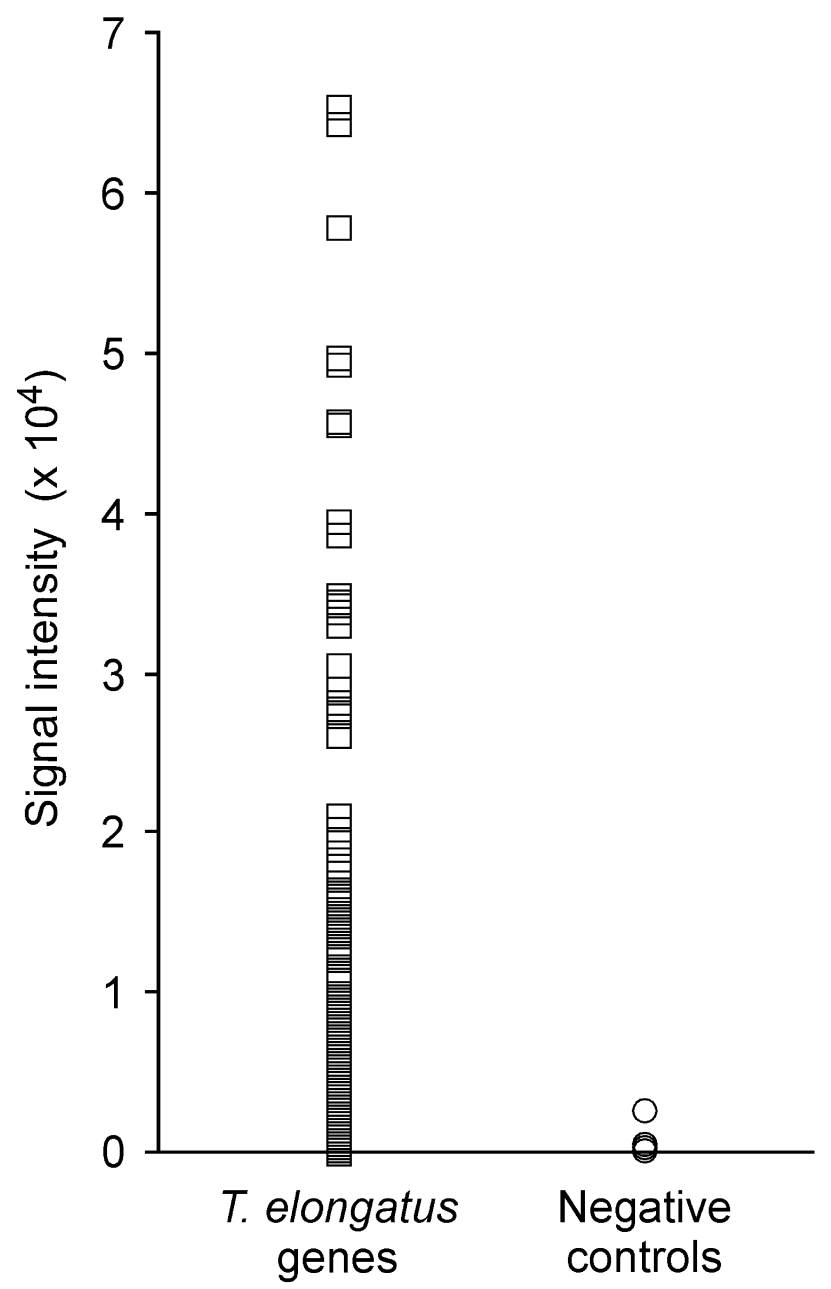

Fig. 2. Signal intensities exhibited by T. elongatus gene probes (open squares) and negative control probes (open circles). A representative result (Cy5-labeled LL14 cDNA) is shown. Seven other hybridization images provided similar results (data not shown).

tion of chaperonin genes is important for adaptation to rising atmospheric temperatures in the daytime. We were surprised, therefore, to find such regulation in a thermophilicorganism that inhabits hot springs.

Genes with expression enhanced in the early subjective night. A large number of genes involved in energy metabolism showed differential expression between LL2 and LL14. Expression of most of them was enhanced in the early subjective night (Fig. 6), suggesting their important role in cyanobacterial physiology. These genes contained 7 subunits of the NADH dehydrogenase complex (ndhA, ndhB, ndhD2, ndhE, ndhH, ndhI, and $n d h K)$ that are involved in respiratory electron transport (Table. 2). Intensive circadian regulation of respiratory genes occurs in Synechocystis (Kucho et al., 2004b), and those genes also show peak expressions in the early subjective night. Such regulation of respiratory genes 


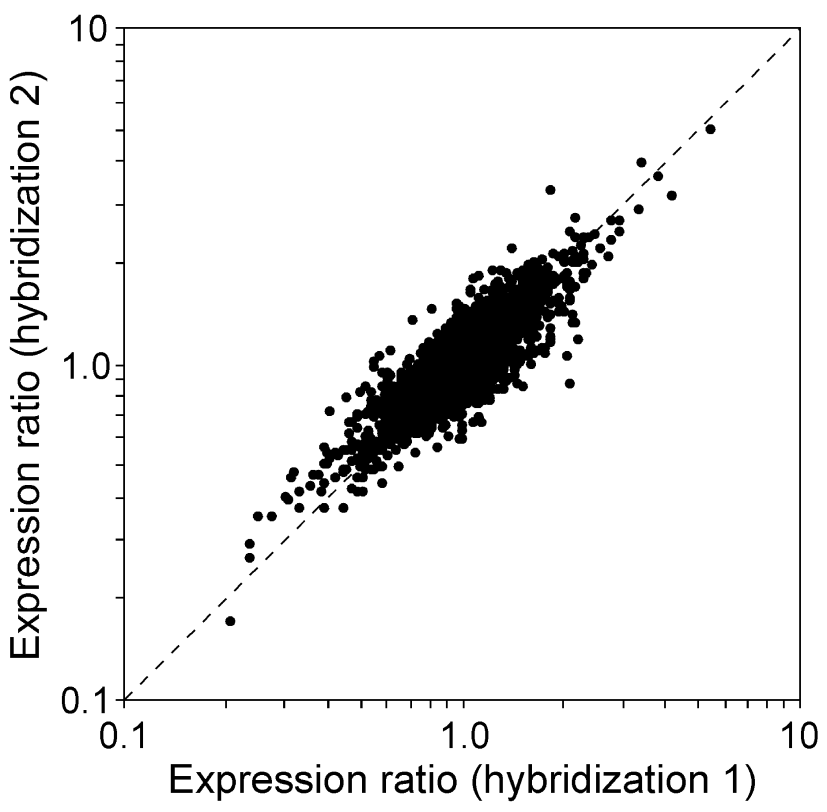

Fig. 3. Reproducibility of the expression ratios (LL14 / LL2) of genes obtained by two independent microarray hybridizations. A representative result is shown. Dashed line represents theoretical values for identical expression ratios.

would contribute to the supply of night-time energy in cyanobacteria. We found that the ftsH gene (tll0734), which is involved in cell division, was activated in the early subjective night (Table 2). In Synechococcus, another cell division gene, ftsZ, also exhibits circadian

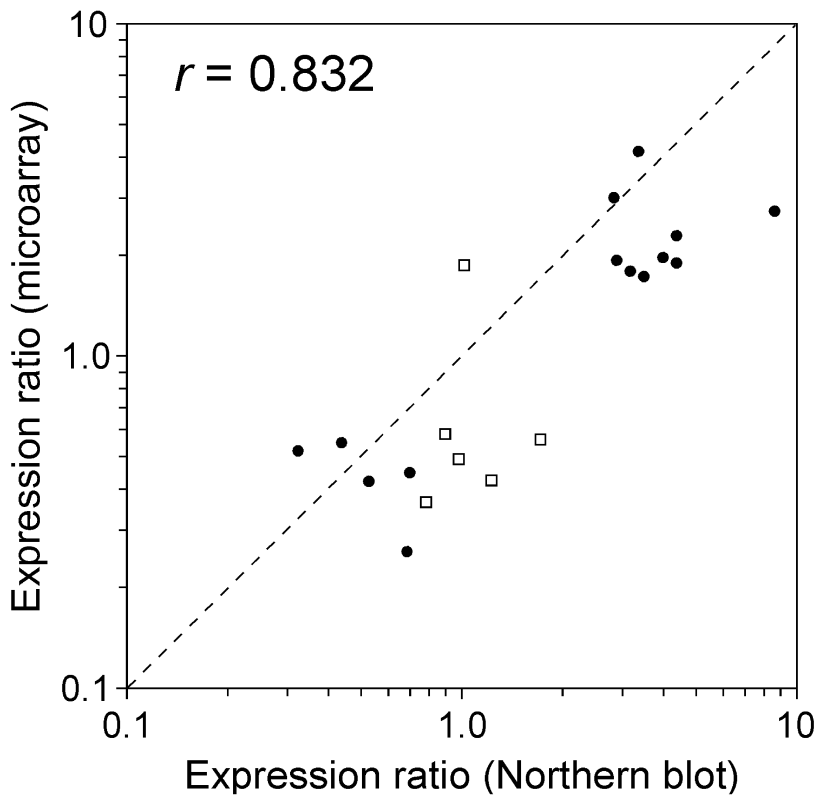

Fig. 5. Comparison of the expression ratios (LL14 / LL2) obtained by microarray and Northern blot analyses. Closed circles represent genes whose expression changes were statistically significant in Northern blot analysis and open squares represent those that were not. The dashed line represents theoretical values for identical expression ratios. $r$ represents correlation coefficient.

rhythm with peak expression in the early subjective night (Mori and Johnson, 2001). The timing of cell division in cyanobacteria is regulated by the circadian clock (Mori et

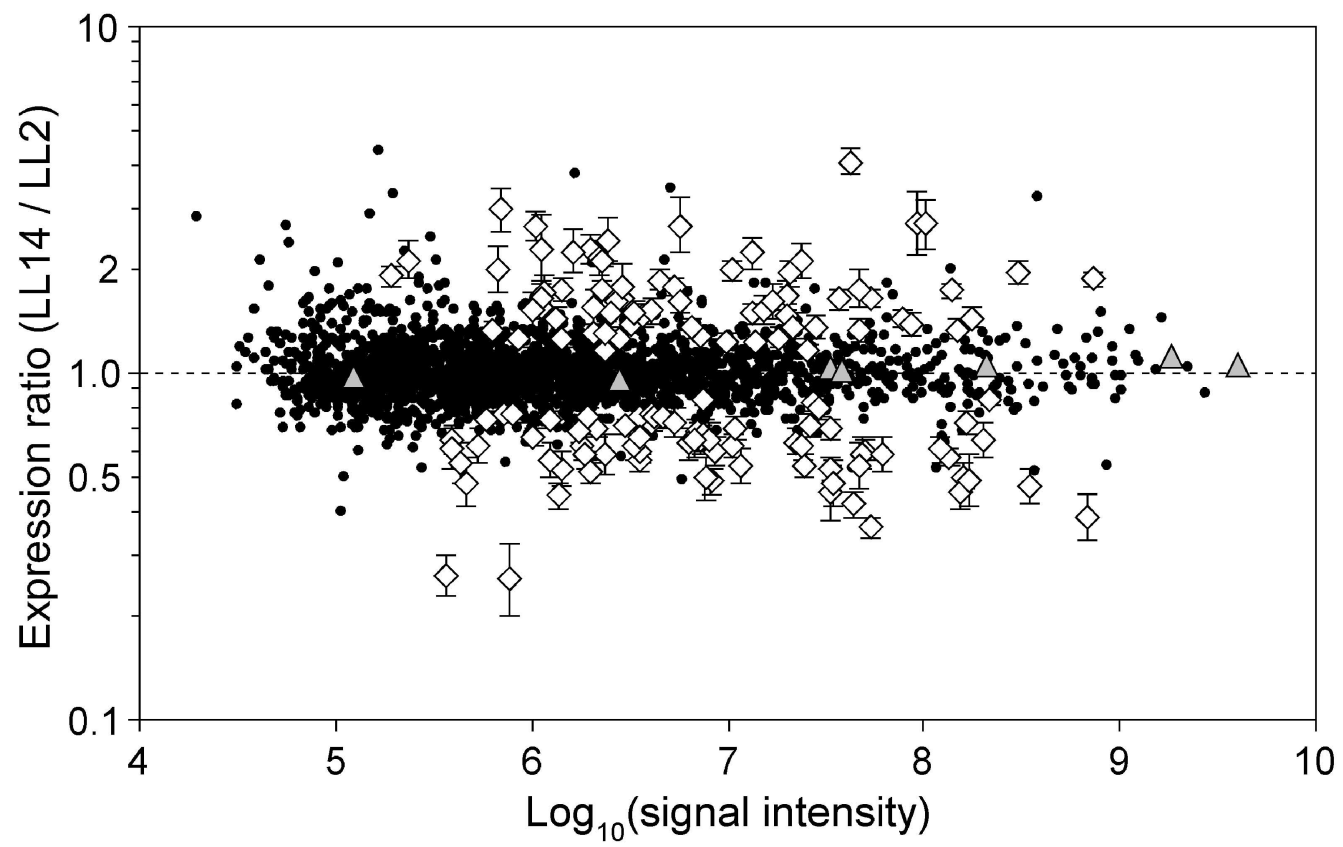

Fig. 4. Comparison of global gene expression in T. elongatus cells between two different circadian times. The $\log _{10}$ of the product of the signal intensities from the two fluorescent dye channels (horizontal axis) roughly corresponds to gene expression level. Open diamonds indicate genes whose expression levels were significantly different between LL2 and LL14. Gray triangles indicate genomic DNA spots. Bars attached to the open diamonds represent the SD for eight measurements at maximum. 


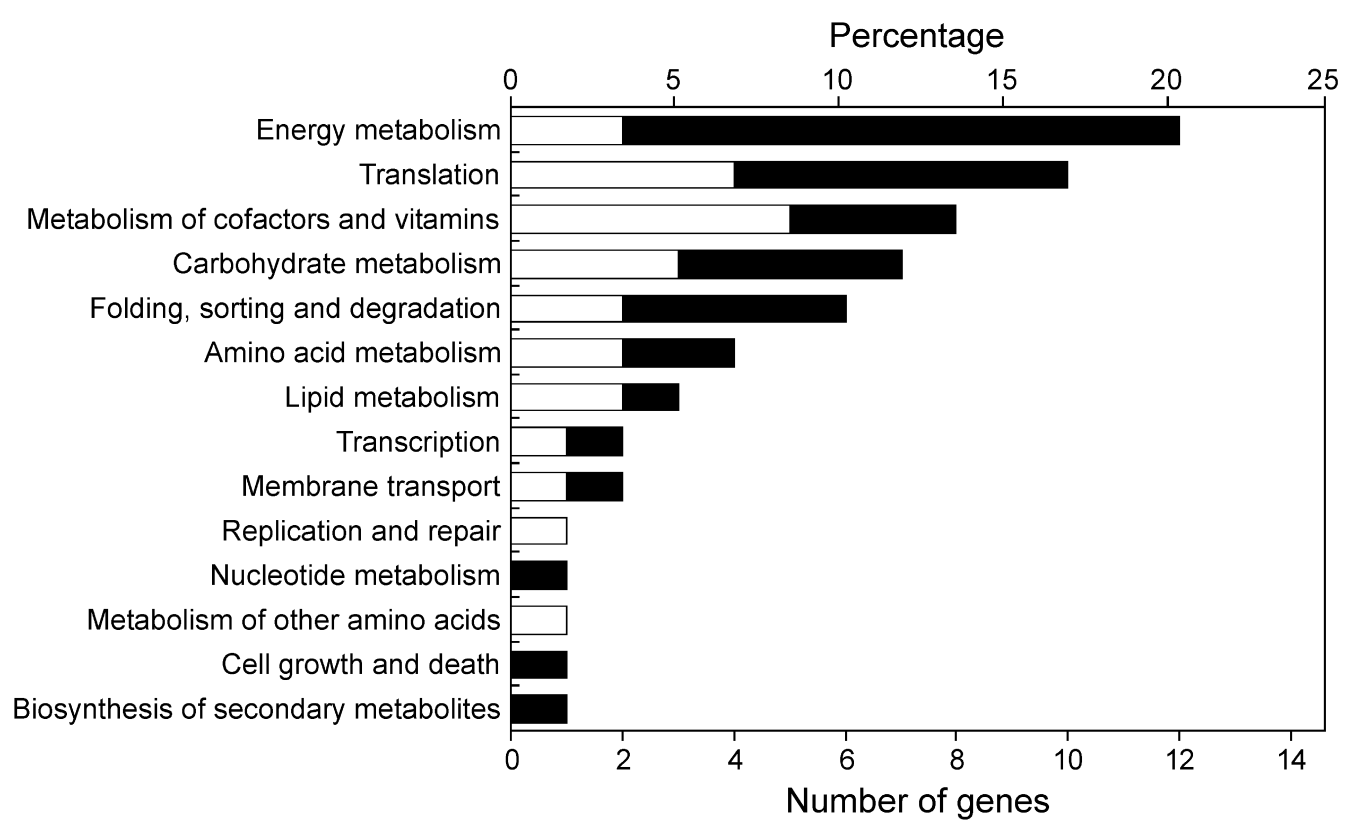

Fig. 6. Percentage of the candidate clock-controlled genes in each functional category. White and black bars represent genes that were activated in the early subjective day and the early subjective night, respectively. Some genes are represented in more than one category.

al., 1996). Circadian regulation of the cell division genes may contribute to the cell division rhythm.

Genes related to translation and transcription. Circadian rhythms in genes related to transcription and translation are of particular interest because quantitative oscillation of those genes could play significant roles in the output mechanism of the circadian clock system. We found that many genes involved in translation showed differential expression between LL2 and LL14 (Fig. 6). They included the elongation factor EF-G (fus: $t l r 1749$ ) and eight ribosomal proteins ( $r p s$ and $r p l$ genes: tll1688, tlr0095, tlr0096, tlr0092, tlr1747, tlr0147, tll1826, and tlr1279) (Tables 1 and 2). Such regulation may yield circadian rhythms at the level of protein synthesis. In the green alga Chlamydomonas reinhardtii, genes encoding chloroplast ribosomal proteins show coordinated circadian rhythm and peak in the early subjective day (K. Kucho, K. Okamoto, S. Tabata, H. Fukuzawa and M. Ishiura, an article submitted). In $T$. elongatus, in contrast, some ribosomal protein genes were activated in the early subjective night while others were activated in the early subjective day (Tables 1 and 2). A gene encoding the $\beta$ subunit of RNA polymerase (rpoB: tll0642) was up-regulated in the early subjective night (Table 2).

In summary, we generated full-genome microarrays of T. elongatus using unmodified oligonucleotide probes. We confirmed the specificity and reproducibility of the hybridization signals and demonstrated the usefulness of the unmodified oligonucleotide-based microarray. This microarray should contribute significantly to the genome biology of thermophilic cyanobacteria, and in addition, allow the comparative genomics of transcriptome between those cyanobacteria for which DNA microarrays are available (such as Synechocystis, Synechococcus and Anabaena sp. PCC 7120). Using the oligonucleotide-based microarray, we identified 143 candidate clock-controlled genes involved in diverse physiological functions. More precise analysis (e.g. at 4-h intervals for $48 \mathrm{~h}$ ) will confirm the circadian status of these genes and provide valuable clues to genome-wide circadian regulation in T. elongatus.

This work was supported by grants from the Japanese Ministry of Education, Science and Culture (MEXT), 'Program for Promotion of Basic Research Activities for Innovative Biosciences' (PROBRAIN) promoted by BRAIN, 'Research for the Future Novel Gene Function Involved in Higher-Order Regulation of Nutrition-Storage in Plants' promoted by the Japan Society for the Promotion of Science, 'Ground-based Research Announcement for Space Utilization' promoted by the Japan Space Forum, 'National Project on Protein Structural and Functional Analyses' promoted by MEXT, and 'Joint-Project for Leading Science and Technology' promoted by the Aichi Science and Technology Foundation to M.I. Division of Biological Science, Graduate School of Science, Nagoya University was supported by a 21st century COE grant from MEXT.

\section{REFERENCES}

Altschul, S. F., Madden, T. L., Schaffer, A. A., Zhang, J., Zhang, Z., Miller, W., and Lipman, D. J. (1997) Gapped BLAST and PSI-BLAST: a new generation of protein database search 
programs. Nucleic Acids Res. 25, 3389-3402.

Aoki, S., Kondo, T., and Ishiura, M. (1995) Circadian expression of the dnaK gene in the cyanobacterium Synechocystis sp. strain PCC 6803. J. Bacteriol. 177, 5606-5611.

Benson, G. (1999) Tandem repeats finder: a program to analyze DNA sequences. Nucleic Acids Res. 27, 573-580.

Hayashi, F., Suzuki, H., Iwase, R., Uzumaki, T., Miyake, A., Shen, J. R., Imada, K., Furukawa, Y., Yonekura, K., Namba, K., and Ishiura, M. (2003) ATP-induced hexameric ring structure of the cyanobacterial circadian clock protein KaiC. Genes Cells 8, 287-296.

Holm, S. (1979) A simple sequentially rejective multiple test procedure. Scand. J. Statis. 6, 65-70.

Ishiura, M., Kutsuna, S., Aoki, S., Iwasaki, H., Andersson, C. R., Tanabe, A., Golden, S. S., Johnson, C. H., and Kondo, T. (1998) Expression of a gene cluster kaiABC as a circadian feedback process in cyanobacteria. Science 281, 1519-1523.

Jordan, P., Fromme, P., Witt, H. T., Klukas, O., Saenger, W., and Krauss, N. (2001) Three-dimensional structure of cyanobacterial photosystem I at $2.5 \AA$ resolution. Nature 411, 909-917.

Kanehisa, M., Goto, S., Kawashima, S., and Nakaya, A. (2002) The KEGG databases at GenomeNet. Nucleic Acids Res. 30, $42-46$.

Kucho, K., Yoneda, H., Harada, M., and Ishiura, M. (2004a) Determinants of sensitivity and specificity in spotted DNA microarrays with unmodified oligonucleotides. Genes Genet. Syst. 79, 189-197.

Kucho, K., Okamoto, K., Tsuchiya, Y., Nomura, S., Nango, M., Kanehisa, M., and Ishiura, M. (2004b) Global analysis of circadian expression in the cyanobacterium Synechocystis sp. strain PCC 6803. J. Bacteriol. in press.

Mori, T., Binder, B., and Johnson, C. H. (1996) Circadian gating of cell division in cyanobacteria growing with average doubling times of less than 24 hours. Proc. Natl. Acad. Sci. USA 93, 10183-10188.

Mori, T., and Johnson, C. H. (2001) Independence of circadian timing from cell division in cyanobacteria. J. Bacteriol. 183, 2439-2444.

Nakamura, Y., Kaneko, T., Sato, S., Ikeuchi, M., Katoh, H., Sasamoto, S., Watanabe, A., Iriguchi, M., Kawashima, K., Kimura, T., Kishida, Y., Kiyokawa, C., Kohara, M., Matsumoto, M., Matsuno, A., Nakazaki, N., Shimpo, S., Sugimoto,
M., Takeuchi, C., Yamada, M., and Tabata, S. (2002) Complete genome structure of the thermophilic cyanobacterium Thermosynechococcus elongatus BP-1. DNA Res. 9, 123130.

Onai, K., Morishita, M., Kaneko, T., Tabata, S., and Ishiura, M. (2004a) Natural transformation of the thermophilic cyanobacterium Thermosynechococcus elongatus BP-1: a simple and efficient method for gene transfer. Mol. Genet. Genomics 271, 50-59.

Onai, K., Morishita, M., Itoh, S., Okamoto, K., and Ishiura, M. (2004b) Circadian rhythms in the thermophilic cyanobacterium Thermosynechococcus elongatus: compensation of period length over a wide temperature range. J. Bacteriol. 186, 4972-4977.

Rippka, R., Deruelles, J., Waterbury, J. B., Herdman, M., and Stanier, R. Y. (1979) Generic assignments, strain histories, and properties of pure cultures of cyanobacteria. J. Gen. Microbiol. 111, 1-61.

Sambrook, J. and Russell, D. W. (2001) Molecular Cloning, third edition. Cold Spring Harbor Laboratory Press, Cold Spring Harbor, New York.

Sanders, D. H. (1995) Statistics: a first course, 5th edition. McGraw-Hill, New York.

Uzumaki, T., Fujita, M., Nakatsu, T., Hayashi, F., Shibata, H., Itoh, N., Kato, H., and Ishiura, M. (2004) Crystal structure of the C-terminal clock-oscillator domain of the cyanobacterial KaiA protein. Nat. Struct. Mol. Biol. 11, 623-631.

Wall, L., Christiansen, T., and Orwant, J. (2002) Programming Perl, third edition. O'Reilly and Associate, CA.

Yamaoka, T., Satoh, K., and Katoh, S. (1978) Photosynthetic activities of a thermophilic blue-green alga. Plant Cell Physiol. 19, 943-954.

Yang, Y. H., Dudoit, S., Luu, P., Lin, D. M., Peng, V., Ngai, J., and Speed, T. P. (2002) Normalization for cDNA microarray data: a robust composite method addressing single and multiple slide systematic variation. Nucleic Acids Res. 30, e15.

Zouni, A., Witt, H. T., Kern, J., Fromme, P., Krauss, N., Saenger, W., and Orth, P. (2001) Crystal structure of photosystem II from Synechococcus elongatus at $3.8 \AA$ resolution. Nature 409, 739-743.

Zuker, M. and Stiegler P. (1981) Optimal computer folding of large RNA sequences using thermodynamics and auxiliary information. Nucleic Acids Res. 9, 133-148. 